\title{
ON SEQUENTIAL HEURISTIC METHODS FOR THE MAXIMUM INDEPENDENT SET PROBLEM
}

\author{
NGOC C. Lê \\ Faculty of Mathematics and Computer Science \\ Technische Universität Bergakademie Freiberg \\ School of Applied Mathematics and Informatics \\ Hanoi University of Science and Technology \\ e-mail: lechingoc@yahoo.com \\ Christoph Brause and Ingo Schiermeyer \\ Faculty of Mathematics and Computer Science \\ Technische Universität Bergakademie Freiberg \\ e-mail: brause@math.tu-freiberg.de \\ ingo.schiermeyer@tu-freiberg.de
}

\begin{abstract}
We consider sequential heuristics methods for the Maximum Independent Set (MIS) problem. Three classical algorithms, VO [11], MIN [12], or MAX [6], are revisited. We combine Algorithm MIN with the $\alpha$-redundant vertex technique [3]. Induced forbidden subgraph sets, under which the algorithms give maximum independent sets, are described. The Caro-Wei bound $[4,14]$ is verified and performance of the algorithms on some special graphs is considered.
\end{abstract}

Keywords: maximum independent set, heuristic, MIN, MAX, VO, vertex ordering.

2010 Mathematics Subject Classification: 05C69, 05C85.

\section{REFERENCES}

[1] V.E. Alekseev and V.V. Lozin, Augmenting graphs for independent sets, Discrete Appl. Math. 145 (2004) 3-10. doi:10.1016/j.dam.2003.09.003 
[2] P. Borowiecki, F. Göring, J. Harant and D. Rautenbach, The potential of greed for independence, J. Graph Theory 71 (2012) 245-259. doi:10.1002/jgt.20644

[3] A. Brandstädt and V.V. Lozin, A note on $\alpha$-redundant vertices in graphs, Discrete Appl. Math. 108 (2001) 301-308. doi:10.1016/S0166-218X(00)00239-0

[4] Y. Caro, New results on the independence, Technical Report, Tel-Aviv University (1979).

[5] M.U. Gerber and V.V. Lozin, On the stable set problem in special $P_{5}$-free graphs, Discrete Appl. Math. 125 (2003) 215-224. doi:10.1016/S0166-218X(01)00321-3

[6] J.R. Griggs, Lower bounds on the independence number in terms of the degrees, J. Combin. Theory Ser. B 34 (1983) 22-39. doi:10.1016/0095-8956(83)90003-5

[7] J. Harant, Z. Ryjáček and I. Schiermeyer, Forbidden subgraphs and MIN-algorithm for independence number, Discrete Math. 256 (2002) 193-201. doi:10.1016/S0012-365X(02)00571-X

[8] N.C. Lê, C. Brause and I. Schiermeyer, New sufficient conditions for $\alpha$-redundant vertices, Discrete Math. 338 (2015) 1674-1680. doi:10.1016/j.disc.2014.07.002

[9] N.C. Lê, C. Brause and I. Schiermeyer, Extending the Max Algorithm for maximum independent set, Discuss. Math. Graph Theory 35 (2015) 365-386. doi:10.7151/dmgt.1811

[10] V.V. Lozin and D. Rautenbach, Some results on graphs without long induced paths, Inform. Process. Lett. 88 (2003) 167-171. doi:10.1016/j.ipl.2003.07.004

[11] N.V.R. Mahadev and B.A. Reed, A note on vertex orders for stability number, J. Graph Theory 30 (1999) 113-120. doi:10.1002/(SICI)1097-0118(199902)30:2〈113::AID-JGT5〉3.0.CO;2-\#

[12] O. Murphy, Lower bounds on the stability number of graphs computed in terms of degrees, Discrete Math. 90 (1991) 207-211. doi:10.1016/0012-365X(91)90357-8

[13] D.J. Rose, R.E. Tarjan and G.S. Lueker, Algorithmic aspects of vertex elimination of graphs, SIAM J. Comput. 5 (1976) 266-283. doi:10.1137/0205021

[14] V.K. Wei, A lower bound on the stability number of a simple graph, Technical Report, Bell Laboratories (1981).

[15] I.E. Zverovich, Minimum degree algorithms for stability number, Discrete Appl. Math. 132 (2004) 211-216. doi:10.1016/S0166-218X(03)00402-5 
Received 29 December 2015

Revised 1 February 2017

Accepted 1 February 2017 